\title{
Las directrices de la jurisprudencia italiana sobre enfermedades profesionales derivadas de la exposición a campos electromagnéticos producidos por teléfonos móviles
}

\author{
Italian Case Law on Occupational Diseases caused by Exposure to \\ Electromagnetic Fields (EMF) generated by Cell Phones
}

\section{LAVINIA SERRANI}

Investigadora ADAPT

lavinia.serrani@adapt.it

https://orcid.org/0000-0003-1116-4521

\author{
MICHELE TIRABOSCHI \\ Catedrático de Derecho del Trabajo \\ Universidad de Módena y Reggio Emilia (Italia) \\ michele.tiraboschi@unimore.it \\ https://orcid.org/0000-0002-6040-0175
}

\begin{abstract}
Resumen: El artículo se centra en el análisis de la jurisprudencia italiana en cuanto al uso del teléfono móvil como causa de aparición de enfermedades profesionales. Los Autores llegan a afirmar que en la actualidad la orientación consolidada de la jurisprudencia parece inclinarse hacia la convicción de que un cierto grado de certeza probabilística pueda considerarse suficiente para reconstruir el nexo causal entre la exposición a ciertos riesgos y la aparición de enfermedades para el reconocimiento de una pensión por enfermedad profesional al trabajador. Según los Autores, esta evidencia confirmaría el retraso de la reflexión de la legislación laboral con respecto al tema de los nuevos riesgos derivados de tecnologías cuyos efectos en las personas se desconocen -mucho más allá del caso del uso de teléfonos móviles y smartphone- con especial referencia a los nuevos entornos de trabajo, los espacios de
\end{abstract}


coworking y la dinámica del trabajo a distancia (el llamado smart working). De hecho, un punto de partida sólido para repensar un sistema de protecciones podría ser fomentar un diálogo interdisciplinario más fructífero entre la investigación médico-científica, la autoridad administrativa y la ciencia jurídica.

Abstract: This paper examines how Italy's case law has dealt with work-related diseases (e.g. acoustic neuroma and parotid gland tumour) caused by cell phone use. In order to award workers sickness benefits, case law has ruled that some degree of probability might be sufficient to establish the causeand-effect relationship between exposure to some risks and the development of a disease. In the authors' view, this aspect confirms that labour laws fail to keep up with the new risks linked to technological innovation, especially in relation to new work environments, co-working areas e remote work. In order to deal with this issue, a starting point could be promoting interdisciplinary cooperation between scientific research, administration bodies and legal sciences.

Palabras claves: jurisprudencia, enfermedades profesionales, campos electromagnéticos, teléfonos moviles.

Keywords: case law, occupational diseases, EMF, cell phones.

Sumario: 1. EXPOSICIÓN A CAMPOS ELECTROMAGNÉTICOS PRODUCIDOS POR TELÉFONOS MÓVILES PARA NECESIDADES LABORALES Y APARICIÓN DE UN TUMOR: CAUSALIDAD JURÍDICA Y PROTECCIÓN LEGAL. 2. ¿UNA ORIENTACIÓN CONSOLIDADA? 3. UN MARCO INCOMPLETO CONDICIONADO POR LAS INCIERTAS RELACIONES ENTRE CIENCIA Y DERECHO. 4. TRANSFORMACIONES LABORALES Y RIESGOS DEBIDOS AL IGNOTO TECNOLÓGICO (TECNOLOGÍAS CUYOS EFECTOS EN LAS PERSONAS SE DESCONOCEN): NUEVOS DESAFÍOS PARA LA PROTECCIÓN LABORAL. 5. BIBLIOGRAFÍA.

\section{EXPOSICIÓN A CAMPOS ELECTROMAGNÉTICOS PRODUCIDOS POR TELÉFONOS MÓVILES PARA NECESIDADES LABORALES Y APARICIÓN DE UN TUMOR: CAUSALIDAD JURÍDICA Y PROTECCIÓN LEGAL}

Con la sentencia del 13 de enero de 2020, n. 904 la Corte di Appello de Turín reconoció la existencia de una relación de causalidad entre la exposición a campos electromagnéticos producidos por teléfonos móviles y la aparición de un neuroma acústico calificado en los términos legales de una enfermedad profesional de etiología multifactorial no reconocida por las instituciones de seguros. La sentencia -que confirma lo ya decidido por el tribunal de primera instancia (Tribunale de Ivrea, sentencia de 30 de marzo de 2017, n. 96)- se refería a un empleado de Telecom Italia S.p.A. quienes, por necesidades de servicio, había hecho un uso anormal (muchas horas al día) y durante un período prolongado (de 1995 a 2010) de los teléfonos antes mencionados. Una vez comprobado, con carga de la prueba impuesta al trabajador y en términos de certeza razonable, el conflicto laboral, la Corte di Appello de Turín 
reiteró en consecuencia la condena del Istituto nazionale per l'Assicurazione Infortuni sul Lavoro (Inail) a pagar al trabajador la pensión relativa por enfermedad profesional.

La decisión de la Corte di Appello de Turín, aunque destacada por la prensa nacional, no es nueva. En todo caso, confirma, al menos como primera aproximación y a la luz de los pocos precedentes disponibles en los repertorios de la jurisprudencia italiana, un enfoque interpretativo que, al menos a primera vista, se está consolidando con el tiempo con respecto a una herramienta (también) de trabajo relativamente nueva como es teléfono móvil que, como tal, sigue siendo difícil de evaluar en términos de riesgos reales o potenciales a medio y largo plazo para la salud de quienes lo utilizan. De hecho, la Corte di Appello de Brescia ya se había expresado en términos similares (sentencia de 10 de diciembre de 2009, n. 361), con una resolución sujeta a acaloradas controversias en el campo médico-legal con respecto a sus supuestos fácticos y científicos tal y como se explicaron al órgano judicial por el asesor técnico de oficio ${ }^{1} \mathrm{y}$, de nuevo en el mismo caso ante decisiones contrarias del Tribunale y de la Corte di Appello de Brescia, también la Corte di Cassazione (sentencia de 12 de octubre de 2012, n. 17438). En la misma línea de apreciación, más recientemente, junto con el mencionado Tribunale de Ivrea, también se expresó el Tribunale de Florencia (sentencia n. 391 de 24 de junio de 2017) $)^{2}$.

Más bien, también para relativizar la resonancia mediática de la resolución, hay que aclarar de inmediato que la sentencia de la Corte di Appello de Turín y todos los precedentes que a la misma se ajustan, no se colocan en la dirección, que pertenece únicamente a las ciencias naturales y médicas, de establecer una conexión cierta y absoluta (la llamada certeza

\footnotetext{
${ }^{1}$ Ver el trabajo de LAGORIO, S., y VECCHIA, P., "Una Corte italiana riconosce l'origine professionale di un neurinoma del trigemino in un utilizzatore di telefoni mobili: un esempio concreto dei complessi rapporti tra scienza e diritto", en La Medicina del Lavoro, 2011, 102, pp. 144-162, y la respuesta de LEVIS, A.G., GRASSO, G., PALMISANO, S., CONSIGLIERE, F., y GENNARO, V., “A proposito dell'articolo su Med. Lav., 2011, 102, 144-162 di S. Lagorio e P. Vecchia. Telefoni mobili e tumori alla testa: la sentenza della Corte d'Appello di Brescia - Sezione Lavoro - alla luce delle attuali conoscenze scientifiche e della legislazione in materia", en La Medicina del Lavoro, 2012, n. 103, pp. 309-313.

2 En doctrina, como comentario a estas sentencias, ver: CASALE, D., "La prova del nesso di causalità professionale per le tecnopatie non tabellate. A proposito della prima pronuncia che riconosce gli effetti cancerogeni dei campi elettromagnetici dei telefoni cellulari”, en $A D L, 2010$, n. 6; VARVARESSOS, A., "Riscontro epidemiologico e probabilità qualificata: nuove prospettive per l'accertamento in concreto dell'elemento causale nelle ipotesi di malattie non tabellate o ad eziologia multifattoriale", en Rivista Italiana di Medicina Legale, 2013, n. 3; ROTA, A., "Sulla natura professionale del tumore contratto per overuse del cellulare in ambito lavorativo", en RIDL, 2013, n. 3; BANORRI, B., "Danni da uso del cellulare e (ir)responsabilità del produttore?", en $R C P, 2013$, n. 4; CERATO, M., y ROMEO, M., "Inquinamento elettromagnetico", en CENDON, P., La prova e il quantum nel risarcimento del danno, Utet, 2013, aquí spec. § 45.5; AL MUREDEN, E., "Uso del cellulare e danni alla salute: la responsabilità del produttore tra dannosità "tollerabile", principio di precauzione e nuovi obblighi informativi", en $C G, 2013$, n. 3; BENINCASA, G., "Il tribunale di Ivrea qualifica come malattia professionale il tumore causato dall'uso scorretto del cellulare", en Boll. ADAPT, 2017, n. 18; PAOLINI, C., "Danni alla salute per uso abnorme del cellulare: si tratta di tecnopatia se sussiste il nesso causale della probabilità qualificata", en $A D L, 2017$, n. 4-5.
} 
determinista) entre el uso del teléfono móvil y la aparición de un tumor. Y esto no solo por el hecho de que, recordando algunos de los casos que han llamado la atención de la jurisprudencia con el propósito de reconstruir el nexo causal entre la exposición a campos electromagnéticos producidos por los teléfonos móviles y la aparición de una patología oncológica, una cosa es el neuroma del nervio acústico (como en el caso tratado por la citada Corte di Appello de Turín) y otra es el tumor de la glándula parótida (como en el caso tratado por el Tribunale de Cremona al que se hará referencia en el § 3). Incluso independientemente de la diversidad y acalorada oposición de opiniones que se registra sobre este punto en la literatura médico-científica ${ }^{3}$, se trata, en retrospectiva, de decisiones relativas, hasta el momento, a figuras profesionales particulares (gerente, coordinador de grupos de trabajo, representante de ventas en una empresa de importación-exportación), en dinámicas de trabajo muy peculiares, si no excepcionales, porque se caracterizan por un uso del teléfono móvil considerado pacíficamente "anormal" y durante un largo período de tiempo, en una época en la que la telefonía móvil aún se encontraba en su etapa embrionaria y el poder adaptativo de las antenas (en ese momento no muy extendido en el territorio) era elevado, y aún no existían dispositivos y herramientas (como auriculares o audífonos) que permitieran evitar la contacto directo del teléfono móvil con el oído y la cara.

Ahora bien, no es que no sea útil resumir (ver infra, § 2), a la luz de los precedentes jurisprudenciales, el estado del arte en cuanto a: 1) riesgo para los trabajadores expuestos a campos electromagnéticos producidos del uso de teléfonos móviles; 2) enfermedades profesionales no reconocidas por las instituciones de seguros, relacionadas con ellas; 3) métodos y técnicas para verificar el vínculo causal (general e individual); 4) espacios y límites para el reconocimiento de una pensión de seguridad social a favor del trabajador a cargo de Inail. Más bien, el examen del razonamiento jurídico avanzado hasta ahora en la materia, tanto en las decisiones de la magistratura como en los comentarios doctrinales que siguieron, debería permitir, sobre todo, poner de relieve los graves retrasos y ciertas lagunas (infra, § 3) que, con respecto a la cuestión del riesgo del llamado ignoto tecnológico (tecnologías cuyos efectos en las personas se desconocen) (infra, § 4), aún confinan la reflexión jurídica (y las protecciones relacionadas) a la única dimensión del daño y la responsabilidad ex post sin una perspectiva evolutiva real, en términos de prevención y evaluación ex ante de los riesgos para la salud y la seguridad de los trabajadores, que vaya más allá de las meras referencias rituales a las disposiciones abstractas (y a menudo ignoradas) de la ley vigente. Esta es una circunstancia que suscita muchas preocupaciones hoy, en medio de una nueva revolución industrial que debería reclamar una atención renovada e incluso diferente de los juristas -y de los juristas laborales en

\footnotetext{
${ }^{3}$ Ver, para todos, las posiciones opuestas, que también se han enfrentado repetidamente en las salas de audiencias, de MOCCALDI, R., y POLICHETTI, A., "Mancato riconoscimento come malattia professionale di un tumore alla parotide in esposto a campi elettromagnetici da telefoni mobili e a radiazioni ionizzanti", en Aggiornamenti di Radioprotezione, 2016, pp. 4-19, por un lado, y LEVIS, A.G., MINICUCI, N., RICCI, P., GENNARO, V., CROSIGNANI, P., y GARBISA, S., "Telefoni mobili e tumori alla testa: è tempo che i dati corretti vengano messi in evidenza e valorizzati?”, en Epidemiologia \& Prevenzione, 2011, pp. 188-199, por otro lado.
} 
particular, si solo se piensa en algunas dolorosas "lecciones del pasado"4- al tema de la protección de la salud y la seguridad en los nuevos entornos de trabajo y debido a las tecnologías de nueva generación introducidas en los procesos productivos ${ }^{5}$. Es cierto, además, que el tema del uso de los teléfonos móviles -por muy común y extendido que sea y, también por ello, objeto de constante interés mediático- es solo la punta del iceberg respecto a las múltiples situaciones, aún no suficientemente monitorizadas, de exposición a campos electromagnéticos en el lugar de trabajo que, debido también a la complejidad objetiva del tema y a la extrema incertidumbre de los estudios médico-científicos realizados hasta ahora, todavía lucha por emerger en el contexto de la reflexión sobre la legislación laboral y la jurisprudencia relacionada (infra, § 3).

\section{2. ¿UNA ORIENTACIÓN CONSOLIDADA?}

A la luz de estas consideraciones introductorias, ahora es posible revisar brevemente y, sobre todo, contextualizar el razonamiento desarrollado por la Corte di Appello de Turín que, en sus pasajes lógicos centrales y en los argumentos técnico-legales relacionados, confirma los escasos precedentes en la materia conocidos en el campo del derecho laboral, por ser publicados y comentados en revistas especializadas.

El punto de partida, al tratarse de enfermedades profesionales no reconocidas por las instituciones de seguros ${ }^{6}$, son los resultados que ofrece la investigación médica y el debate científico que, como se sabe, aún no han llegado a una conclusión compartida sobre las consecuencias (nocivas o no) para la salud de las personas por el uso de teléfonos móviles ${ }^{7}$. En

\footnotetext{
${ }^{4}$ Véase, en particular, TULLINI, P., “A rischio amianto?”, en RIDL, 2007, I, aquí p. 455, con referencia a un caso, tratado principalmente en términos coyunturales, como el del amianto.

${ }^{5}$ Sobre este tema, para una visión general del problema, véase el extenso informe presentado por la Comisión al Parlamento, al Consejo y al Comité Económico y Social Europeo sobre Safety and Liability Implications of Artificial Intelligence, the Internet of Things and Robotics, 19 de febrero de 2020, COM(2020)64 final.

${ }^{6}$ En lo que respecta a las enfermedades profesionales, en Italia hay una serie de casos, las llamadas "malattie tabellate", en las que existe una presunción legal sobre su origen laboral, es decir, determinada por las condiciones laborales o el trabajo realizado. El listado de enfermedades en cuestión es exhaustivo, a diferencia de las enfermedades "non tabellate" en las que no opera la presunción legal y se requiere que el trabajador demuestre la existencia real de la enfermedad, las características de las funciones realizadas, que sean tales que provoquen la aparición de la enfermedad y relación causal entre la enfermedad y el cargo que ocupa. Sobre el tema, para la reconstrucción del sistema de cobertura de seguros italiano, ver GIUBBONI, S., LUDOVICO, G., Y ROSSI, A., Infortuni sul lavoro e malattie professionali, Cedam, 2014, cap. III, § 10, y también CERBONE, M., Il giudice e l'Inail nell'applicazione delle norme sulle malattie professionali, Working Papers Olympus, 2014, n. 34.

${ }^{7}$ Una extensa reconstrucción del debate científico y del estado de la investigación se puede encontrar, con respecto a los limitados perfiles especializados que son relevantes a efectos de un análisis de derecho laboral, en los precedentes jurisprudenciales mencionados anteriormente a los que se añade la bibliografía citada en GOBBA, F., MODENESE, A., ZANOTTI, G., "Effetti dei campi elettromagnetici nei lavoratori professionalmente esposti: le evidenze scientifiche, le decisioni della magistratura", en GOLDONI, S., CONTESSA, G.M., NATALETTI, P., DELLA VECCHIA, N., y NICOLINI, O., Radiazioni ionizzanti e non ionizzanti: valutazione e protezione alla luce della nuova normativa europea, Modena, 2017, pp. 140-141, disponible en acceso abierto en la library de la plataforma SALUS, en la dirección http://salus.adapt.it.
} 
realidad, no faltan estudios sobre radiofrecuencias que excluyen decididamente cualquier vínculo causal entre el uso de teléfonos móviles y los tumores cerebrales. Sin embargo, se trata de análisis y valoraciones que, aunque formulados en el ámbito científico, no son considerados fiables por la magistratura (al menos la conocida por los abogados laboralistas) debido a la posición de conflicto de intereses en la que se encontrarían involucrados sus autores (como consultores de operadores de telefonía celular o empresas del sector) o, en todo caso, por el carácter no público de la financiación que (también gracias a las aportaciones económicas de las empresas telefónicas) permitió realizar la investigación. La Corte di Appello de Turín escribe: "(es evidente que) la investigación y las conclusiones de autores independientes dan mayores garantías de fiabilidad que las encargadas, gestionadas o financiadas, al menos en parte, por sujetos interesados en el resultado de los estudios. [...] Además, precisamente en una disputa contra Inail relacionada con una enfermedad profesional (tumor intracraneal) por exposición a radiofrecuencia desde un teléfono móvil, la Suprema Corte di Cassazione sostuvo que "El énfasis adicional en la mayor confiabilidad de estos estudios, dada su posición de independencia, es decir, por no haber sido cofinanciados, a diferencia de otros, incluso por los propios fabricantes de teléfonos celulares, constituye una adicional y no ilógica base de las conclusiones aceptadas" (ver Corte di Cassazione 12.10.2012, n. 17438)"».

La ausencia de certeza absoluta en el campo de la investigación científica y médica sobre el peligro o no de los teléfonos celulares para la salud de las personas no afecta, sin embargo, el razonamiento jurídico para la protección del empleado, dado que la magistratura no se coloca en la perspectiva de la existencia o no de una ley científica. Lo que importa más bien, a los efectos y dentro de los límites de una valoración jurídica en términos de riesgo profesional y daño, es precisamente la ausencia de certezas y, si acaso, la presencia de sospechas y dudas que luego abren el camino a lo probable y posible ${ }^{9}$. Esto permite a los magistrados formular, en términos generales y a la luz del principio de precaución (infra, § 4), una evaluación de la posible nocividad de las ondas electromagnéticas emitidas por los teléfonos móviles (y no solo por ellos: infra, § 3) con respecto a los cuales los resultados de experimentos con animales y diversos estudios estadístico-epidemiológicos independientes, aunque no del todo concordantes, indicarían la existencia, en ausencia de factores de riesgo alternativos y en circunstancias particulares relacionadas con la edad, cantidad y duración de la exposición al factor de riesgo, un grado suficiente de certeza probabilística a los efectos de reconstruir el

\footnotetext{
${ }^{8}$ Sobre el tema, para una denuncia que va más allá de los límites de nuestras competencias científicas y técnicas, pero que sin embargo es útil para señalar la delicadeza del perfil de independencia real y neutralidad de la investigación que se está haciendo sobre los efectos de los campos electromagnéticos en la salud. de personas, ver también LEVIS, A.G., GRASSO, G., PALMISANO, S., CONSIGLIERE, F. y GENNARO, V., A proposito dell'articolo su Med. Lav., 2011, 102, 144-162 di S. Lagorio e P. Vecchia. Telefoni mobili e tumori alla testa ecc., cit., e ivi, p. 313, también la respuesta de Susanna Lagorio e Paolo Vecchia.

${ }^{9}$ En general, ver VISCOMI, A., “Amianto: precauzione, prevenzione e responsabilità”, en MONTUSCHI, L., e INSOLERA, G., Il rischio amianto. Questioni sulla responsabilità civile e penale, Bononia University Press, 2006, aquí p. 54.
} 
vínculo causal válido para la ley ${ }^{10}$ y para la aplicación, en el caso concreto, de las consecuencias jurídicas relacionadas, es decir, en nuestro caso, el reconocimiento de una pensión por enfermedad profesional. Esta es una situación que la magistratura enmarca técnicamente en los términos de un "riesgo relativo", es decir, una medida probabilística del aumento del riesgo causado por la exposición al factor presuntamente patógeno, mediante el uso de la expresión "más probable que no"11.

En la reconstrucción sistemática del problema causal en los contextos de causas concomitantes y eventos multifactoriales -como es el caso que es objeto de nuestra reflexión y como es la situación imperante en la actualidad por la propagación de enfermedades crónico-degenerativas y enfermedades neoplásicas atribuibles a factores de riesgo ubicuo, a los que se puede estar expuestos incluso fuera del lugar de trabajo ${ }^{12}$ - el aspecto del vínculo causal jurídico y el perfil probatorio están en tal medida interconectados "que parecen casi epifenómenos de la misma causa, que es la identificación de una metodología de investigación por casos, a la cual un razonamiento (nomo)lógico-deductivo es inaplicable"13. Razonando de esta manera, el nexo causal puede por tanto considerarse probado a partir de las encuestas estadísticas documentadas por estudios epidemiológicos sobre los efectos de la exposición a campos electromagnéticos producidos por teléfonos móviles, obviamente contextualizados a la peculiaridad y dinámica del caso específico de cáncer y del historial laboral individual en cuestión ${ }^{14}$, aunque la ciencia médica aún no haya llegado a definir y compartir la existencia de una ley científica real ${ }^{15}$.

\footnotetext{
${ }^{10}$ Así: Cass. 12 de octubre de 2012, n. 17438, e ivi más referencias jurisprudenciales. En docrtina, ver: ROMEO, L., "Nesso di causalità nelle malattie multifattoriali", en RIMP, 2016, aquí p. 322.

${ }^{11}$ Ver., entre muchas, Cass. 27 de abril de 2004, n. 2073, en RGL, 2005, II, pp. 199-210, con nota de SACCONI, G., La prova del nesso di causalità nelle malattie multifattoriali: l'importanza del criterio epidemiologico. Sobre el tema, para la evolución del concepto de causalidad con referencia específica a la relación entre causalidad general y causalidad individual, ver, para todos, FIORI, A., "La causalità nelle malattie professionali", en Atti del VI Convegno nazionale di medicina legale previdenziale, S. Margherita di Pula 18-20 ottobre 2006, Inail, 2006, p. 36 y spec. pp. 42-50, donde se concreta el propium de la valoración probabilístico-inductiva en el uso de criterios cronológicos, topográficos, cuantitativos, de continuidad, clínico-anamnésicos y sobre todo de exclusión de otras causas. Ver. también SFERRA, C., Confronto tra malattia professionale non tabellata e malattia comune, en $R I M P, 2010$, aquí spec. pp. 479-483, y MANCA, G., "Assalti e difese ai bastioni della causalità scientifica nei contributi più recenti di dottrina e giurisprudenza", en $R C P, 2013$, pp. 476-503.

${ }^{12}$ En estos términos, DE MATTEIS, A., "La prova della malattia multifattoriale. L'equivoco continua", en RIMP, 2014, aquí p. 583.

${ }^{13}$ Así: VARVARESSOS, A., Riscontro epidemiologico e probabilità qualificata ecc., cit., aquí p. 1559.

${ }^{14}$ Ver de nuevo VARVARESSOS, A., Riscontro epidemiologico e probabilità qualificata ecc., cit., aquí p. 1560.

${ }^{15}$ En este sentido (entre las muchas decisiones, incluso inéditas, recordadas en el amplio estudio editado por LEVIS, A.G., La posizione innovativa della Magistratura Italiana sui rischi per la salute da CEM, 2012, disponible open access en la library de la plataforma SALUS, cit.) ver: Cass. n. 9893/2000; Trib. Modena n. 1430/2004; Trib. Venezia n. 441/2008; Cass. Pen. n. 33285/2008; App. Milano n. 2168/2009, Cass. n. 23676/2009; Cass., sez. un., n. 581/2008; Cass. n. 15991/2011; Cass. n. 3227/2011.
} 
En esta perspectiva de análisis, como especifica la Corte di Appello de Turín siguiendo la enseñanza constante de la jurisprudencia de la legitimidad (ver, entre muchas otras, Cass.10 de abril de 2018, n. 8773) "al tratarse de una enfermedad profesional de etiología multifactorial no reconocida por las instituciones de seguros, el cargo de la prueba en el procedimiento judicial, sin duda impuesto al trabajador, [...] debe valorarse en términos de certeza razonable y, por tanto, excluyendo la relevancia de la mera posibilidad de origen profesional, se puede reconocer en presencia de un grado significativo de probabilidad". Esto se basa en los resultados ofrecidos, para cada caso, no solo por el trabajador, sino también por los asesores médico legales nombrados de oficio (ver Corte di Cassazione 12 de octubre de 2012, n. 17438, y Tribunale de Florencia 24 de junio 2017, n. 391), también debido a las tareas realmente realizadas por el trabajador, el tipo específico de enfermedad y las características concretas (en términos de duración e intensidad) de la exposición del trabajador a las radiofrecuencias.

Como especifica la Corte di Cassazione (sentencia de 24 de noviembre de 2015, n. 23951) "en la hipótesis de una enfermedad de etiología multifactorial -como el cáncer- el nexo causal relativo a su origen profesional no puede ser objeto de simples presunciones extraídas de hipótesis técnicas teóricamente posibles, sino que requiere una demostración concreta y específica, que incluso puede darse como probabilidad, pero solo en el caso de "probabilidad calificada", la cual necesita ser verificada a través de elementos adicionales adecuados para traducir en certeza jurídica las conclusiones en términos probabilísticos del asesor técnico". Y esto cuando en los casos concretos, una vez excluida la relevancia de la mera posibilidad de procedencia profesional (Corte di Cassazione 10 de febrero de 2011, no. 3227), se identifican elementos y datos que no justifican, en términos precisamente probabilísticos, una exclusión del nexo causal entre la exposición a campos electromagnéticos y la aparición de la enfermedad. Una "certeza razonable", en definitiva, suficiente para que la magistratura detecte los extremos del nexo causal jurídico y, en consecuencia, permita el reconocimiento a favor del trabajador de una pensión por enfermedad profesional, aunque esta no esté incluida en las tablas de las instituciones de seguros (en los mismos términos ver Corte di Cassazione 12 de octubre de 2012, n. 17438). Se trata de una causalidad jurídica todavía individual (y no meramente general) y que, sin embargo, también puede verificarse con respecto a una "ley" estadísticoepidemiológica: "es decir, no se trata de comparar los resultados de un estudio sobre una muestra de casos clínicos relacionados con la patología en cuestión con el caso concreto, sino de considerar válidos, incluso frente a estudios adicionales o alternativos, aquellos que presentan variables de evaluación también subsistentes y relevantes en el caso concreto y que permitan una demostración lógica a partir de una simple comparación con el índice de riesgo reconocido en la materia ${ }^{16}$.

\footnotetext{
16 Así, siempre con referencia a Cass. 12 de octubre de 2012, n. 17438, VARVARESSOS, A., Riscontro epidemiologico e probabilità qualificata ecc., cit., aquí p. 1564. Ver también, en general, COGGIOLA, N., "Il giudice e la statistica: attività lavorative, esposizione all'amianto ed asbestosi ovvero quando il numero di morti e malati fa la prova nel proceso", en GI, 2005, pp. 1172-1176.
} 
Finalmente, en esta línea de análisis, queda por precisar, a partir de lo señalado por el Tribunale de Florencia (sentencia de 24 de junio de 2017), que la circunstancia "de que probablemente el teléfono móvil también haya sido utilizado por motivos personales y por lo cual no puede constituir una causa exclusiva de la patología, sin embargo, no excluiría la relación concausal con la actividad laboral, teniendo en cuenta que, por consolidada orientación jurisprudencial, en materia de accidentes de trabajo y enfermedades profesionales, se aplica la regla contenida en el artículo 41 del código penal, en virtud del cual la relación de causalidad entre hecho y daño se rige por el principio de equivalencia de condiciones, según el cual debe reconocerse la eficiencia causal de cualquier antecedente que haya contribuido, incluso de manera indirecta y remota, a la producción del evento, a menos que el nexo etiológico sea interrumpido por la ocurrencia de un factor suficiente por sí solo para producir el evento, con el efecto de degradar las causas precedentes a simples ocasiones" (en este sentido, ver también Corte di Cassazione 19 de junio de 2014, núm. 13954, y Corte di Cassazione 9 de septiembre de 2005, núm. 17959, al que se refiere en este punto el Tribunale de Florencia).

\section{UN MARCO INCOMPLETO CONDICIONADO POR LAS INCIERTAS RELACIONES ENTRE CIENCIA Y DERECHO}

Las consideraciones desarrolladas hasta ahora abren inevitablemente el camino a muchos problemas jurídicos. Sobre todo, el relativo a la elección -no obligatoria pero que, sin duda, representa la vía principal en decisiones como las objeto de nuestro comentario- del asesor técnico judicial que, como es fácilmente comprensible, dada la complejidad de los reconocimientos médicos respecto a un panorama bastante variado y controvertido de opiniones e investigaciones científicas, juega un papel decisivo en la formación de la convicción, por parte del juez, sobre la existencia o no del nexo causal jurídico. La atribución al magistrado de la tarea de resolver en última instancia, sobre la base de los resultados de las asesorías técnicas solicitadas a instancia de parte y de oficio, cuestiones científicas que no son fáciles de evaluar ni siquiera entre los expertos, es un tema muy conocido, que durante mucho tiempo ha sido objeto de atención por la reflexión jurídica tanto en procesos civiles como penales. Con los recientes avances en el conocimiento científico, en paralelo con la introducción en los procesos productivos y en el lugar de trabajo de tecnologías de nueva generación que multiplican las fuentes de riesgo y las posibles enfermedades profesionales, cabe preguntarse si los jueces -al menos considerando la formación y las habilidades que tienen hoy, que son el resultado de las vías ordinarias de entrenamiento y selección- puedan ser los verdaderos gatekeepers de la fiabilidad del conocimiento especializado y de las evaluaciones científicas que entran en el proceso $^{17}$. Si este enfoque podía "parecer razonable mientras las cuestiones técnicas de interés para la ley eran relativamente simples y comprensibles incluso para un "laico" como el juez",

\footnotetext{
${ }^{17}$ Para esta terminología, que recuerda los lineamientos de la jurisprudencia norteamericana, ver ya MASERA, L., "Giudice e perito nel processo penale: spunti per un dibattito", en Epidemiologia e Prevenzione, 2005, aquí p. 305.
} 
va hoy recibiendo cada vez más crédito la duda sobre la razonabilidad y sostenibilidad de la opción de confiar a una persona que carece (al menos por la forma en que se organiza hoy la selección y formación de los magistrados) de cualquier competencia médico-científica la decisión sobre temas altamente especializados y complejos incluso para expertos en la materia: “¿cómo podemos creer que juez, quién hasta el momento del juicio tal vez nunca había oído hablar de una ciencia llamada epidemiología [...] sea milagrosamente capaz de adquirir habilidades que le permitan resolver racionalmente el conflicto entre los expertos de las distintas partes del juicio?"18. Aunque centrales -y de hecho destinadas a aumentar en importancia e intensidad con respecto a la cada vez más frenética e incierta persecución entre la innovación tecnológica, las evaluaciones científicas de nocividad y la regulación legal de los fenómenos económicos- las cuestiones 1) de la validez de las vías actuales de selección y formación de los magistrados, 2) de los criterios para verificar la calificación profesional y la "neutralidad" del asesor técnico de oficio e incluso 3) de la posibilidad de encomendar a sujetos con habilidades especializadas adecuadas la legitimidad para emitir valoraciones médicocientíficas vinculantes para el juez en el juicio, inevitablemente van más allá de los límites de esta contribución ${ }^{19}$.

Respecto a los lineamientos de la magistratura destacados hasta ahora, aparentemente $\operatorname{consolidados}^{20}$, queda por subrayar un aspecto crítico adicional aún no destacado por la

${ }^{18}$ Así MASERA, L., Giudice e perito nel processo penale ecc., cit., p. 305. En los mismos términos también TARUFFO, M., "Le prove scientifiche nella recente esperienza statunitense", en RTDPC, 1996, pp. 219-249, y SALOMONE, E., "Sulla motivazione con riferimento alla consulenza tecnica d'ufficio", en RTDPC, 2002, aquí p. 1026. Contra, entre muchos: RADOS, B. y GIANNINI, P., La consulenza tecnica nel processo civile, Giuffrè, 2013, aquí p. 41. Sobre el tema, ver también BOVE, M., "Il sapere tecnico nel processo civile", en RDP, 2011, pp. 1431-1450.

19 Aquí podemos limitarnos a señalar, para el primero de estos tres puntos críticos, la posibilidad de una combinación entre un título de tres años en medicina básica y un master en derecho o viceversa. Así, respecto a la necesidad de una mayor interdisciplinariedad en la construcción de las profesiones jurídicas, ver la propuesta de ENRIQUES, L., "Come si forma un avvocato", en Lavoce.info, 26 de septiembre de 2013. Sobre el tema, ver también RIVERSO, R., "Dalla tutela del lavoratore alla tutela della persona. Il profilo oggettivo", en RIMP, 2015, spec. p. 416, donde recuerda como "el artículo 202 del Testo Unico del 1965 prevé cursos especialización -nunca activados que se sepa- a cargo de Inail para jueces en materia de accidentes de trabajo y enfermedades profesionales". Por otro lado, con respecto a la cuestión de la calificación profesional y de los criterios para la elección del asesor técnico de oficio, claramente analizados ya por el Tribunale de Como (orden de 30 de noviembre de 2001, spec. $\S 6.3$ de la motivación, en relación con el papel del asesor técnico de oficio en la verificación del vínculo causal entre la leucemia infantil y la exposición a campos electromagnéticos producidos por dos líneas de conducción eléctrica de alta tensión), ver ZANETTI, R., "Il sapere scientifico in tribunale", en Epidemiologia e Prevenzione, 2005, pp. 307-309; TRENTA, G., "Competenze e professionalità nelle consulenze d'ufficio in tribunale", en La Medicina del Lavoro, 2011, 102, pp. 163-166 y también GOBBA, F., MODENESE, A., y ZANOTTI, G., Effetti dei campi elettromagnetici nei lavoratori professionalmente esposti: le evidenze scientifiche, le decisioni della magistratura, cit., spec. p. 138, donde critican algunas de las sentencias analizadas en el párrafo anterior debido a que, según ellos, se basan en conclusiones de los asesores técnicos de oficio, los cuales se habrían desviado de las orientaciones predominantes en la comunidad científica. En cuanto a la posibilidad de encomendar a terceros calificados valoraciones médico-científicas vinculantes para el juez, ver MASERA, L., Giudice e perito nel processo penale ecc., cit., p. 306.

${ }^{20}$ En este sentido, ver PAOLINI, C., Danni alla salute per uso abnorme del cellulare ecc., cit., p. 1285. 
reflexión jurídica que se ha medido sobre la controvertida relación entre exposición a campos electromagnéticos y protección de la salud de los trabajadores. Nos referimos a la ausencia en nuestro país de una base de datos que contenga todas las sentencias, incluso de mérito, existentes sobre el tema. Como ha señalado una fuente médico-científica ${ }^{21}$, la percepción de los juristas respecto a las orientaciones de la magistratura está quizás parcialmente distorsionada por una suerte de cortocircuito comunicativo: "Los medios (de hecho) informaron solo noticias relativas a algunas sentencias que se pronunciaron a favor de la existencia de un nexo causal, pero en realidad en otros procesos la magistratura se expresó en sentido contrario", donde el conocimiento de la existencia de varios casos judiciales con diferentes conclusiones podría brindar una visión más integral y menos distorsionada del problema en cuestión a los profesionales, la opinión pública y los responsables políticos. En otras palabras, "la difusión de noticias relativas únicamente a los procesos concluidos con el reconocimiento de una relación causal entre exposición ocupacional a CEM y cáncer induce una situación, comparable a la que en el ámbito científico se define "publication bias", que hace una hipótesis más creíble, aunque solo sea porque parece ser la única posible"22.

Y, de hecho, una compleja investigación empírica, basada en la información fragmentaria disponible en la literatura médico-científica ${ }^{23}$, también indica la existencia de precedentes de signo contrario a los hasta ahora recordados en el debate sobre derecho laboral, al ser éstos los únicos que se reportan en los repertorios de jurisprudencia y en las revistas especializadas. También en este caso, nos enfrentamos, en realidad, con un número limitado de decisiones. Suficiente, sin embargo, por un lado, para poner en duda la existencia de una orientación verdaderamente inequívoca de la magistratura y, por otro, para volver a proponer en el centro de la reflexión jurídica el problema de la centralidad del asesor técnico con respecto a la adhesión o no a una orientación alarmista con respecto a las conexiones, que varían también según los sectores de producción y los tipos de procesamiento (véase, en general, el informe Inail sobre Tumori professionali: analisi per comparti economici, 2019), entre la exposición a campos electromagnéticos producidos por teléfonos móviles y la aparición de un tumor. Sin olvidar que, en los repertorios de la magistratura, existe un silencio casi total no solo sobre gran parte de los cánceres que podrían tener origen profesional, sino también respecto a otras posibles enfermedades profesionales no reconocidas por las instituciones de seguros vinculadas a la exposición a campos electromagnéticos que se investigan, con diversidad de voces y opiniones, en la literatura médico-científica al menos con referencia a algunas enfermedades neurodegenerativas y cardiovasculares y otras patologías que afectan el sistema reproductivo,

\footnotetext{
21 Ver GOBBA, F., MODENESE, A. y ZANOTTI, G., Effetti dei campi elettromagnetici nei lavoratori professionalmente esposti: le evidenze scientifiche, le decisioni della magistratura, cit., aquí p. 138.

${ }^{22}$ Otra vez GOBBA, F., MODENESE, A. y ZANOTTI, G., op. loc. ult. cit.

${ }^{23}$ Véanse en particular los casos citados en MOCCALDI, R. y POLICHETTI, A., Mancato riconoscimento come malattia professionale di un tumore alla parotide in esposto a campi elettromagnetici da telefoni mobili e a radiazioni ionizzanti, cit.
} 
el sistema inmunológico-neuroendocrino, así como los posibles efectos neuroconductuales y otros vinculados al bienestar de los trabajadores ${ }^{24}$.

En la literatura médico-científica ${ }^{25}$ también se ha destacado, más recientemente, el fenómeno de la llamada hipersensibilidad a los campos electromagnéticos ${ }^{26}$ que afectaría a un porcentaje limitado, pero no despreciable, de la población con efectos que varían significativamente de persona a persona. Los síntomas más frecuentes son dermatológicos (enrojecimiento, picor, sensación de ardor), neuróticos y vegetativos (fatiga, cansancio, insomnio, dificultad para concentrarse, náuseas, dolores de cabeza, sensación de inestabilidad, palpitaciones, alteraciones digestivas). La jurisprudencia francesa se ha ocupado recientemente de estos problemas, con referencia a un técnico expuesto a las ondas electromagnéticas emitidas en el lugar de trabajo por un espectrómetro de masas isotópico. En este caso, el Tribunal Administrativo de CergyPontoise (sentencia de 17 de enero de 2019, n. 1608265) no dudó en reconocer, por primera vez en Francia, los requisitos de una enfermedad profesional: "en dépit de l'absence de consensus médical, en l'état des connaissances scientifiques, sur un lien de causalité entre les effets à long terme sur la santé et l'exposition aux champs électromagnétiques, dans les circonstances particulières de l'espèce et, notamment, en présence d'un avis favorable de la commission de réforme, au constat d'une exposition sur le lieu de travail prolongée, significative, plurielle et simultanée à des champs électromagnétiques de fréquences multiples, dont certains atypiques, aux troubles réels et invalidants médicalement constatés développés par M. F. deux ans après sa prise de fonction et, enfin, à l'absence d'état antérieur ou de facteurs extérieurs aux conditions particulières d'exécution de son service, il existe une probabilité suffisante que l'hypersensibilité électromagnétique contractée par le requérant soit en rapport avec son activité professionnelle. M. F. est dès lors fondé à soutenir que la décision contestée du 1er juillet 2016 portant refus d'imputabilité au service de son syndrome d'hypersensibilité électromagnétique est entachée d'une erreur d'appréciation et à en demander, pour ce motif, l'annulation".

Todo esto especificado, de extrema relevancia, al menos con respecto al tema de nuestras notas sobre el nexo causal jurídico entre la exposición a campos electromagnéticos producidos por teléfonos celulares (y otros equipos de trabajo como un medidor de densidad instalado en la línea de producción) y la aparición de un tumor, son las sentencias (no registradas hasta ahora

\footnotetext{
${ }^{24}$ Para una revisión de los datos ofrecidos por la literatura especializada, ver GOBBA, F., MODENESE, A. y ZANOTTI, G, Effetti dei campi elettromagnetici nei lavoratori professionalmente esposti: le evidenze scientifiche, le decisioni della magistratura, cit., p. 135.

25 Ver GRECO, M., "Inquinamento elettromagnetico e salute fra controversie, ricerca indipendente ed associazionismo Il caso del movimento degli elettrosensibili”, en EtnoAntropologia, 2016, pp. 119-150.

${ }^{26}$ Sobre el tema, ver ya la World Health Organization, Campi elettromagnetici e salute pubblica. Ipersensibilità ai campi magnetici, diciembre 2005.
} 
en los repertorios de la jurisprudencia italiana) del Tribunale de Cremona y del Tribunale de Milán.

La sentencia del Tribunale de Cremona (sentencia de 10 de abril de 2015, n. 39) se destaca, en primer lugar, por la adhesión a una metodología médico-legal para el reconocimiento del nexo causal en el campo de la exposición a campos electromagnéticos con base en una evaluación consecuencial de la causalidad (de general a individual) realizada a través del criterio de la llamada "probability of causation" que deberá determinarse mediante evidencia científica consolidada, es decir, posibilidad científica y probabilidad estadística ${ }^{27}$. En esta perspectiva también se explica el intento de recusación, por parte de los abogados del trabajador, de los dos asesores técnicos designados por el Tribunal, por estar notoriamente posicionados, en el debate científico sobre el tema, a favor de la metodología que niega la existencia de evidencias científicas que respalde la tesis de un nexo causal entre el uso de teléfonos móviles y la aparición de tumores (en este caso se trataba de un carcinoma de parótida). Recusación que, aunque rechazada por el Tribunal (orden de 28 de abril de 2014), vuelve a poner de relieve la centralidad, en los procesos judiciales en esta materia, del asesor técnico de oficio y de sus convicciones médico-científicas. Más aún en este caso concreto, donde son cuarenta las páginas especializadas que los asesores técnicos de oficio han dedicado, en su informe final (en gran parte recogido entre comillas en el texto de la sentencia), a la cuestión del nexo causal entre uso de teléfonos móviles por motivos de servicio y aparición de carcinoma parietal ${ }^{28}$. Otro punto de interés es la referencia al precedente reiteradamente mencionado de la Corte di Appello de Brescia (sentencia de 10 de diciembre de 2009, n. 361), que, en consonancia con las observaciones de los asesores técnicos de oficio, el Tribunale de Cremona no considera relevante para el caso concreto. Por un lado, porque se trata de una decisión previa a la evaluación del Centro Internacional de Investigaciones sobre el Cáncer en $2011^{29}$ que, como informa la sentencia del Tribunale de Cremona, "clasificó los campos electromagnéticos emitidos por los teléfonos móviles como "posiblemente carcinógenos para el ser humano" (Grupo 2B del sistema de clasificación IARC), decidiendo así no clasificarlos como "carcinógenos para el ser humano" (Grupo 1 del sistema de clasificación IARC) o como "agentes probablemente carcinógenos para el ser humano" (Grupo 2A del sistema de clasificación IARC)". Como se sabe, la clasificación en el Grupo 2B significa que estamos en presencia de "agentes para los que existen pruebas limitadas de carcinogenicidad en humanos y pruebas insuficientes de carcinogenicidad en experimentación animal" ${ }^{30}$. Por otro lado,

\footnotetext{
${ }^{27}$ Ver MOCCALDI y R., POLICHETTI, A., Mancato riconoscimento come malattia professionale di un tumore alla parotide in esposto a campi elettromagnetici da telefoni mobili e a radiazioni ionizzanti, cit., aquí p. 5.

28 Sobre este aspecto ver en detalle la contribución de MOCCALDI, R., y POLICHETTI, A., Mancato riconoscimento come malattia professionale di un tumore alla parotide ecc., cit., pp. 4-20.

${ }^{29}$ IARC, Non-ionizing radiation, part 2: radiofrequency electromagnetic fields, 2013, vol. 102, aquí p. 419, disponible open access en la library de la plataforma SALUS, cit.

${ }^{30}$ IARC, Non-ionizing radiation, part 2: radiofrequency electromagnetic fields, cit., aquí p. 30.
} 
porque, nuevamente para el Tribunale de Cremona de acuerdo con las conclusiones alcanzadas por los asesores técnicos nombrados a petición de las partes, "la valoración de causalidad general requiere que la idoneidad lesiva en cuestión se demuestre a través de evidencias científicas consolidadas, las cuales deberían buscarse más en la literatura científica que en la jurisprudencia". En otras palabras, prosigue el Tribunale de Cremona, "para poder afirmar la existencia de un nexo causal entre el uso de teléfonos móviles por parte del actor y el tumor de parótida que lo afectó, habría sido necesario encontrar en la literatura científica evidencia consolidada de que el uso de teléfonos móviles es generalmente adecuado para determinar el tumor de parótida (evaluación causal general y criterio de idoneidad lesiva)".

En la misma línea argumental centrada en el sistema de clasificación de la IARC, considerado "hasta la fecha el estudio más completo y autorizado en la literatura científica sobre la correlación causal entre la exposición a campos electromagnéticos de RF y la aparición de un tumor", se posicionó, más recientemente, también el Tribunale de Milán (sentencia n. 959 de 31 de julio de 2018) con referencia a un caso de uso intensivo y diario del teléfono móvil por un trabajador con las funciones de "Asistente Superior" y luego de "Responsable del Departamento de Compras", durante un largo período de tiempo. También en esta circunstancia, de manera similar a lo ocurrido en el precedente de Cremona, el Tribunal primero desestimó (orden de 18 de julio de 2017) el recurso de recusación de los asesores técnicos designados de oficio interpuesto por el trabajador y luego consideró irrelevante la decisión contraria del Tribunale de Ivrea (sentencia de 30 de marzo de 2017, n. 96), recordada por los abogados del trabajador, debido a la no demostración en el presente caso, relativo a un tumor cerebral (oligodendroglioma grado II opercular frontal derecho), del nexo de causalidad o concausalidad entre la exposición mencionada a campos electromagnéticos emitidos por teléfonos móviles y la aparición de la patología neoplásica cerebral diagnosticada al trabajador, es decir, resultando no cumplido, a la luz de la evidencia científica sobre el tema y en términos de probabilidad calificada, "el criterio de idoneidad lesiva y que, por tanto, el oligodendroglioma diagnosticado no puede ser considerado, con un alto grado de probabilidad, causalmente vinculado a su exposición a estos campos, independientemente del alcance de la exposición". Al no estar probada la derivación profesional de la enfermedad, también en este segundo caso se rechaza la solicitud del trabajador de una pensión por enfermedad profesional a cargo de Inail.

Se trata de precedentes que aún no han entrado en el debate laboral sobre la materia objeto de nuestras consideraciones y que, sin embargo, parecen suficientes para cuestionar la existencia de una orientación consolidada en la jurisprudencia, señalándose, en todo caso, como la probable punta del iceberg con respecto a las múltiples situaciones de riesgo por exposición a 
campos electromagnéticos presentes en el lugar de trabajo ${ }^{31}$ y que, poco a poco, van entrando en las salas de audiencias.

Al respecto, de hecho podemos señalar, nuevamente entre los precedentes inéditos, una sentencia de la Corte di Appello de Perugia (sentencia de 9 de enero de 2012, n. 473) que negó el nexo causal con respecto a la exposición de un trabajador de Telecom (y, anteriormente, empleado de la Empresa Estatal de Servicios Telefónicos - ASST y, luego, de Intel S.p.A.), posteriormente fallecido de cáncer, a los campos electromagnéticos generados por las antenas para la recepción de señales de radiofrecuencia. Para la Corte di Appello de Perugia, de hecho, hay una falta de "estudios que lleven a la conclusión o al menos a sospechar una posible acción oncogénica con respecto a los tumores sólidos de la nariz y de los senos paranasales, en particular del etmoides, por exposición a radiofrecuencias" donde, con respecto al tipo de tumor objeto de la controversia, "la mitad de los casos observados a nivel mundial involucran a sujetos que, por motivos profesionales, se vieron expuestos a la inhalación de polvo de madera y, en menor medida, del cuero; además, los trabajadores del sector textil, los trabajadores involucrados en la fusión y soldadura de metales, los trabajadores expuestos a polvos de níquel, cromo y formaldehído se consideran en riesgo. Por otro lado, no se ha demostrado claramente una acción oncogénica del humo del tabaco". En resumen, "como señaló el asesor técnico de oficio, actualmente no existe evidencia científica de que la exposición a ondas electromagnéticas pueda ocasionar neoplasias, y en particular carcinoma del etmoides, que afectó al cónyuge del recurrente y causó su muerte".

En la misma perspectiva, se observa una sentencia reciente de la Corte di Cassazione (sentencia de 11 de julio de 2019, n. 18701) que excluyó la correlación causal entre la neoplasia diagnosticada en la víctima (técnico de mantenimiento de Enel) y la exposición a campos electromagnéticos o aceites que contienen PCB. Sobre la base de la instrucción realizada y del informe del asesor técnico de oficio, la Corte di Appello de Reggio Calabria (sentencia de 8 de enero de 2014) ya había descartado que las funciones realizadas por el trabajador en cuestión hubieran implicado una exposición a campos electromagnéticos (o aceites que contienen PCB) de tal intensidad y frecuencia que puedan desempeñar un papel causal o concausal en la aparición de la patología hepática y aplasia medular y, posteriormente, de la neoplasia de lengua que provocó su muerte. La Corte di Cassazione, al confirmar la sentencia de la Corte di Appello, precisó que "el juez competente no está obligado a justificar plenamente las razones de su adhesión a las conclusiones del asesor técnico de oficio, si no existen argumentos en contra de las partes o estos no son específicos, pudiendo, en este caso, limitarse a reconocer aquellas conclusiones como justificadas por las investigaciones realizadas por el experto y por las explicaciones contenidas en el respectivo informe (ver, ex plurimis, Corte di Cassazione n. 1660 de 2014; n. 25862 de 2011; n. 10688 de 2008; n. 4797 de 2007; n. 26694 de 2006; n. 10668 de

\footnotetext{
${ }^{31}$ Véase ya CAMPURRA, G., Il rischio da campi elettromagnetici negli ambienti di lavoro, Ipsoa, 2008.
} 
2005); en todo caso, constituye un principio firme el según el cual el defecto -denunciable en la etapa en que se evalúa la legitimidad- de la sentencia que se ha adherido a las conclusiones del dictamen pericial forense, solo puede comprobarse en el caso de una clara desviación de las nociones actuales de la ciencia médica, cuya fuente debe ser indicada en la omisión de valoraciones instrumentales que según las nociones antes mencionadas no pueden ser despreciadas para la formulación de un diagnóstico correcto, mientras que fuera de este contexto la censura constituye un mero disenso diagnóstico que termina representando una crítica inadmisible a la condena del juez (cfr. ex multis Corte di Cassazione n. 1652 del 2012; n. 569 del 2011; n. 9988 del 2009)".

Desde el punto de vista del derecho, la Corte di Cassazione confirma linealmente lo que sostiene la citada sentencia de la Corte di Appello de Turín y es que, "en el caso de una enfermedad, como el cáncer, de etiología multifactorial, la prueba del nexo causal no puede consistir en simples presunciones inferidas de hipótesis técnicas teóricamente posibles, sino que debe consistir en la demostración concreta y específica, al menos en términos de probabilidad, de la idoneidad de la exposición al riesgo para causar el evento morboso (entre otros ver Corte di Cassazione n. 23653 de 2016), por lo que todavía es necesario que sea una "probabilidad calificada", a ser verificada a través de elementos adicionales adecuados para traducir la conclusión probabilística en certeza judicial (ver, entre muchos, Corte di Cassazione n. 9057 de 2004 y, más recientemente, Corte di Cassazione n. 10097 de 2015; Corte di Cassazione n. 13814 de 2017)". Sin embargo, las conclusiones sobre el fondo son opuestas debido a las diferentes opiniones expresadas en las respectivas fases (Corte di Appello de Turín y Corte di Appello de Reggio Calabria) por el consultor técnico de oficio que, una vez más, resultan decisivas a los efectos de verificación del nexo causal jurídico.

Confirman esta impresión, de un diálogo aún no lineal entre el análisis médico-científico y el análisis jurisprudencial, dos precedentes más de signo contrario (y hasta donde sabemos inéditos) del Tribunale de Verona y del Tribunale de Monza donde el reconocimiento por parte del juez del nexo causal entre la exposición a campos electromagnéticos y la aparición de un tumor siempre se produce en fiel adhesión a los resultados de la investigación realizada por el asesor técnico y a la literatura científica introducida y acreditada por él en el proceso. El caso tramitado por el Tribunale de Verona (sentencia n. 293 de 7 de junio de 2017) se refería a un trabajador asignado a la explotación de una depuradora de aguas residuales civiles e industriales, el cual debía realizar, durante un número significativo de horas al día, actividades de monitoreo en un lugar de trabajo que contenía (debajo del piso) numerosos cables de conducción eléctrica y transformadores eléctricos que generaban campos electromagnéticos de baja frecuencia. Como leemos textualmente en la sentencia, es el asesor técnico de oficio quien, tras "una escrupulosa, consecuencial y minuciosa investigación médico-legal, [...] llega a la conclusión de la existencia de un nexo etiológico entre la exposición a campos electromagnéticos de baja frecuencia (radiación no ionizante) y la aparición de trombocitemia. [...] Según el asesor técnico de oficio, no existe una ley de cobertura científica por la cual, al eliminar mentalmente la exposición a radiaciones no ionizantes, se llegaría con una alta 
probabilidad a excluir la patología (el llamado criterio de "eliminación mental", desarrollado por la doctrina del derecho penal), sin embargo es razonablemente posible que el recurrente, sobre la base de su historial clínico y laboral, finalmente haya contraído la enfermedad en cuestión como consecuencia de la exposición a lo largo de los años. El nexo causal así aclarado, además de ser compartido por este juez, lleva a considerar integrada la prueba del nexo causal entre la enfermedad observada y el trabajo realizado, ya que no es necesario un alto grado de verosimilitud (como habría requerido, en cambio, una investigación criminal sobre la responsabilidad del empleador)". El Tribunale de Verona es consciente de que la investigación realizada hasta el momento sobre el tema "no demuestra un nexo en términos de certeza o alta probabilidad, sino solo de posibilidad". Por tanto, es plausible en abstracto, prosigue el Tribunal, que "una exposición a radiaciones no ionizantes provoque la aparición de enfermedades graves". Pues bien, una vez aclarada esta premisa, concluye el Tribunal, "el paso siguiente implica el estudio, en detalle, de la exposición ocupacional y el análisis de la historia clínica y profesional del recurrente. $\mathrm{Y}$ es precisamente el informe del perito, junto con la investigación técnica y documental de la instrucción, lo que despeja el campo de una posible etiopatogenia alternativa, apoyando finalmente de forma adecuada y reflexiva la tesis del recurrente".

En los mismos términos se expresó el Tribunale de Monza (sentencia n. 56 de 13 de marzo de 2019) con referencia a un agente de pista en los aeropuertos de Linate y Malpensa (coordinador de operaciones de carga y descarga y repostaje) expuesto en el desempeño de sus funciones a las frecuencias emitidas por numerosas antenas y dispositivos de radiocomunicación de alta frecuencia, por repetidores de señales radioaltimétricas, por radares meteorológicos y antenas satelitales, por otros equipos instalados en la plataforma de tráfico y por otras ondas electromagnéticas producidas por 10 teléfonos celulares GSM activos, conectados y cargándose en la red eléctrica, 5 dispositivos portátiles a cargar, 2 computadoras personales encendidas constantemente, 2 repetidores de señal, un teléfono celular para comunicaciones de servicio y numerosos otros equipos que lo exponían durante más de cuatro horas al día a ondas electromagnéticas de muy alta frecuencia. A raíz del resultado del informe encomendado al asesor técnico de oficio, realizado sobre la base de la clasificación del Centro Internacional de Investigaciones sobre el Cáncer (CIIC) de los campos electromagnéticos de radiofrecuencia y los resultados sobre los factores de riesgo presentes en el caso en cuestión, el Tribunal llega a la conclusión que, con referencia a la aparición de neuroma acústico en el trabajador, no se pueden reconocer otros posibles agentes etiopatogenéticos preponderantes. Aun admitiendo, a la luz de la literatura científica sobre el tema, una dificultad significativa en cuanto al reconocimiento de una correlación causal entre la exposición a radiofrecuencias y la aparición de patologías tumorales, el juez, adhiriéndose fielmente al informe del asesor técnico de oficio y recordando el precedente de la Corte di Appello de Brescia (sentencia de 10 de diciembre de 2009, n. 361), considera en el caso en cuestión la existencia de un papel al menos concausal entre la aparición del neuroma acústico y el trabajo realizado por el recurrente también debido a la duración e intensidad de la exposición. Y por estas razones condena a Inail a pagar al trabajador la pensión relativa por enfermedad profesional. 


\section{TRANSFORMACIONES LABORALES Y RIESGOS DEBIDOS AL IGNOTO TECNOLÓGICO (TECNOLOGÍAS CUYOS EFECTOS EN LAS PERSONAS SE DESCONOCEN): NUEVOS DESAFÍOS PARA LA PROTECCIÓN LABORAL}

Las reflexiones realizadas hasta ahora sobre las orientaciones inciertas y no transparentes -en términos de acceso al conocimiento, al menos si pensamos en el enorme potencial de la big data analytics aplicada a los contenidos de las sentencias de todo orden y grado- de la magistratura, respecto a la existencia de un nexo causal entre la exposición a campos electromagnéticos en el lugar de trabajo y la aparición de una enfermedad profesional, confirman el retraso de la reflexión de la legislación laboral italiana con respecto al tema de los nuevos riesgos derivados de tecnologías cuyos efectos en las personas se desconocen, mucho más allá del caso del uso de teléfonos móviles y smartphone en el lugar de trabajo. En cuanto a los nuevos entornos laborales, a los espacios de coworking y a la dinámica del trabajo a distancia (el llamado smart working), aún no se ha desarrollado una reflexión en el ámbito del derecho laboral $^{32}$ sobre el impacto en la salud y seguridad de trabajadores del $5 \mathrm{G}$, de las redes WiFi, de la carga sin contacto de TIC móviles y uso de interfaces cerebrales ${ }^{33}$.

Sin embargo, una lección se puede extraer del examen de la jurisprudencia realizado en los párrafos anteriores. Y es la de iniciar cuanto antes un diálogo interdisciplinario diferente y más fructífero entre la investigación médico-científica, las autoridades administrativas y las respuestas del ordenamiento jurídico a la exposición a una fuente de riesgo como los campos electromagnéticos, anticipándose a la fase relativa a la redacción del estándar normativo (que es tarea de la política legislativa) y su implementación concreta en la dinámica de las estructuras organizativas del trabajo y vigilancia médica ${ }^{34}$, análisis prejudiciales (sobre la peligrosidad o no de determinadas herramientas, productos o tecnologías para la salud de los trabajadores) que no sólo pueden confiarse a las valoraciones de intereses y a los dolorosos hechos humanos que entran en el proceso una vez que el daño ya haya ocurrido.

\footnotetext{
${ }^{32}$ A excepción de una mención fugaz en el documento final realizada por el European Risk Observatory, Foresight on new and emerging occupational safety and health risks associated with digitalisation by 2025, European Agency for Safety and Health at Work, 2018, aquí p. 55.

${ }^{33}$ Sobre el tema, véanse las cuestiones críticas planteadas recientemente por el extenso Rapporto indipendente sui campi elettromagnetici e diffusione del $5 G$ de septiembre de 2019 promovido por ISDE y European Consumers al que se agrega el Appeal for 5G Moratorium - Scientists and doctors warn of potential serious health effects of 5G, September 13, 2017.

${ }^{34}$ En lo que véase, con respecto al tema de nuestras notas, BELLIA, M., CAMPURRA, G., DE LUCA, G., GIOVANAZZI, A., GOBBA, F., L'ABBATE, N., LODI, V., MALESANI, F., MOCCALDI, R., OTTENGA, F., PENNAROLA, R., PERSECHINO, B., RIGHI, E., STANGA, A. y TRENTA, G., Linee Guida AIRM Sorveglianza medica dei lavoratori esposti a radiazioni ionizzanti, Ipsoa-Wolters Kluwer, 2013.
} 
Por un lado, se trata de repensar desde los cimientos, en una perspectiva renovada de law and economics como lo decía Calabresi ${ }^{35}$, la teoría jurídica del riesgo y el concepto mismo de riesgo empresarial en la dimensión de la IV revolución industrial. Esto se debe a las relaciones cada vez más entrelazadas e inextricables entre productor, empresario-usuario y trabajadorproductor-consumidor, al menos con respecto a cuestiones legales y contractuales que se extienden en gran medida más allá de los estrechos confines de los (antiguos) lugares de trabajo y del control del empleador, para plantear cuestiones de salud pública, incluso antes que la salud ocupacional $^{36}$, que van más allá incluso de los estrechos espacios de viabilidad de la responsabilidad civil del productor $^{37}$ y que plantean a las autoridades públicas cuestiones delicadas de risk assessment y de introducción de obligaciones de divulgación reforzadas con respecto a los riesgos asociados con la comercialización y el uso de ciertas tecnologías en el lugar de trabajo ${ }^{38}$. Una perspectiva, esta, que lleva a dudar, en materia de salud pública y a la luz de las más recientes transformaciones en el trabajo, no solo del tradicional planteamiento jurídico de tipo compensatorio, sino también de la plena pertinencia de las disposiciones contenidas en la Directiva 2013/35/UE de 26 de junio de 2013 (recientemente transpuesta al ordenamiento jurídico italiano con el decreto legislativo n. 159 de 1 de agosto de 2016). Ciertamente no con referencia a las normas contenidas en la misma en términos de límites de exposición y niveles de acción ${ }^{39}$, sino porque todavía se concibe y desarrolla con referencia a contextos de trabajo tradicionales y de evaluación de los riesgos relacionados.

Por otro lado, se trata de ir más allá de los límites de una evaluación ex post, de forma puramente compensatoria, de las consecuencias de las actividades peligrosas para la salud y seguridad de los trabajadores, recuperando, incluso en los modelos organizativos impuestos por la IV

\footnotetext{
${ }^{35}$ CALABRESI, G., The Costs of Accidents, Yale University Press, 1970, autor, como se sabe, atento no solo al perfil de eficiencia en la asignación de costos y responsabilidades, sino también al problema de la justicia social, sobre el que véanse en particular las reflexiones contenidas en las pp. 24-26 y pp. 301-308.

${ }^{36}$ En estos términos ver, para algunas ideas reconstructivas importantes, LEROUGE, L., "Réflexions juridiques autour du rapport "Santé au travaul: vers un systéme simplifié pour une prévention rendorcée"”, en DS, 2019, pp. 151-159.

${ }^{37}$ En particular la responsabilidad ex artículo 2050 código civil, sobre la cual véase, para todos, AL MUREDEN, E., "I danni da utilizzo del cellulare tra conformità del prodotto agli standard legislativi, principio di precauzione e autoresponsabilità dell'utilizzatore", en $C G, 2017$, n. 8-9, que resalta cómo el principio de precaución se dirige al legislador y a las autoridades administrativas, mientras que no asume efecto directo en las relaciones entre particulares.

${ }^{38}$ En esta perspectiva, véase el informe presentado por la Comisión al Parlamento, al Consejo y al Comité Económico y Social Europeo sobre Safety and Liability Implications of Artificial Intelligence, the Internet of Things and Robotics, cit.

${ }^{39}$ Problema sobre el que no tenemos competencia para expresar una valoración técnica de mérito y respecto al cual nos limitamos a referir las dudas planteadas, en general, en la doctrina por VISCOMI, A., Amianto: precauzione, prevenzione e responsabilità, cit., aquí pp. 46-47, quien, recordando las conocidas posiciones sobre el tema del magistrado italiano Roberto Riverso, habla de los valores umbral en términos de lotería dolorosa. Sobre el tema, es obligatoria la remisión a BECK, U., La società del rischio, Carocci, 2013 pero 1986, aquí pp. 84-92 donde habla del "engaño de los valores máximos permitidos".
} 
revolución industrial, esa dimensión preventiva que está claramente delineada en nuestra Constitución y en la legislación vigente en esta materia ${ }^{40}$. Sin embargo, en nuestra opinión, sigue siendo central la construcción en el mercado laboral de renovadas figuras profesionales "híbridas", es decir, con habilidades técnico-profesionales adecuadas para gestionar los nuevos riesgos $^{41} \mathrm{y}$, desde el punto de vista jurídico, capaces de asegurar una mayor adherencia en el lugar de trabajo entre disposiciones legales abstractas y comportamientos concretos de empleadores y trabajadores de acuerdo con los principios de innovación y desarrollo tecnológico pero también con los de precaución en la gestión de tecnologías, herramientas o aparatos cuyos efectos sobre la salud y la seguridad de los trabajadores aún no se conocen con certeza $^{42}$.

Después de todo, si la historia realmente enseña algo, sería "simplemente" extender a los riesgos que se derivan de unas tecnologías cuyos efectos en las personas todavía se desconocen, ese rico "patrimonio de conocimientos y experiencias" acumulado a través de la larga y dolorosa evolución de la legislación y jurisprudencia en materia de amianto. Porque "incluso el riesgo desconocido (o supuestamente tal) se puede prevenir, si el sistema preventivo funciona y el empleador cumple sin reservas la obligación de seguridad, utilizando el sentido común y la diligencia profesional ordinaria" ${ }^{\natural 3}$, más aún hoy en medio de una nueva revolución industrial.

\section{BIBLIOGRAFÍA}

AL MUREDEN, E., "I danni da utilizzo del cellulare tra conformità del prodotto agli standard legislativi, principio di precauzione e autoresponsabilità dell'utilizzatore", en Il Corriere Giuridico, 2017.

\footnotetext{
${ }^{40}$ Ver, para todos, RIVERSO, R., Dalla tutela del lavoratore alla tutela della persona ecc., cit., aquí p. 413, y también GIUBBONI, S., LUDOVICO, G., ROSSI, A., Infortuni sul lavoro e malattie professionali, cit., aquí pp. 23-24, que muestran cómo "la mera prestación de servicios económicos no puede considerarse suficiente -si no a modo de subrogación- para realizar la garantía constitucionalmente asegurada”.

${ }^{41}$ Con referencia a los campos electromagnéticos, véanse las notas críticas de BIAZZI, L., "La nuova direttiva di radioprotezione e il punto di vista dell'esperto qualificato nella sua applicazione in campo industriale, sanitario e di ricerca", EN GOLDONI, S., CONTESSA, G.M., NATALETTI, P., DELLA VECCHIA, N., y NICOLINI, O., Radiazioni ionizzanti e non ionizzanti: valutazione e protezione alla luce della nuova normativa europea, cit., spec. p. 26, donde aborda la cuestión del contraste entre la figura presente en nuestra tradición del experto cualificado y el perfil del radiation protection expert al que se refiere la directiva 2013/59/EURATOM.

${ }^{42}$ Sobre el tema ver, para todos, PATRIZI, E., Il principio di precauzione nella società del rischio, tesis doctoral, Universidad de Macerata, 2014, passim y cap. II, § 2.5 por los riesgos de contaminación electromagnética, disponible open access en la library de la plataforma SALUS, cit.

43 Así, efectivamente, MONTUSCHI, L., "Il rischio amianto: quale tutela? Introduzione al dialogo", en MONTUSCHI, L., y INSOLERA, G., Il rischio amianto ecc., cit., aquí p. 10, al que se agrega TULLINI, P., A rischio amianto?, cit., aquí p. 455.
} 
AL MUREDEN, E., "Uso del cellulare e danni alla salute: la responsabilità del produttore tra dannosità "tollerabile", principio di precauzione e nuovi obblighi informativi", en Il Corriere Giuridico, núm. 3, 2013.

BANORRI, B., “Danni da uso del cellulare e (ir)responsabilità del produttore?”, en $R C P$, núm. 4., 2013.

BECK, U., La società del rischio, Carocci, Roma, 2013, pero 1986.

BELliA, M., CAMPURRA, G., DE LUCA, G., GIOVANAZZI, A., GOBBA, F., L'ABBATE, N., LODI, V., MALESANI, F., MOCCALDI, R., OTTENGA, F., PENNAROLA, R., PERSECHINO, B., RIGHI, E., STANGA, A. y TRENTA, G., Linee Guida AIRM Sorveglianza medica dei lavoratori esposti a radiazioni ionizzanti, Ipsoa-Wolters Kluwer, 2013.

BENINCASA, G., "Il tribunale di Ivrea qualifica come malattia professionale il tumore causato dall'uso scorretto del cellulare", en Boll. ADAPT, núm., 18., 2017.

BIAZZI, L., "La nuova direttiva di radioprotezione e il punto di vista dell'esperto qualificato nella sua applicazione in campo industriale, sanitario e di ricerca", en GOLDONI, S., CONTESSA, G.M., NATALETTI, P., DELLA VECCHIA, N., y NICOLINI, O., Radiazioni ionizzanti $e$ non ionizzanti: valutazione $e$ protezione alla luce della nuova normativa europea, Modena, dBA, 2017, pp. 13-30, disponible en acceso abierto en la library de la plataforma SALUS, en la dirección http://salus.adapt.it.

BOVE, M., "Il sapere tecnico nel processo civile”, en RDP, núm. 6., 2011.

CALABRESI, G., The Costs of Accidents: a legal and economic analysis, Yale University Press, New Haven, 1970.

CAMPURRA, G., Il rischio da campi elettromagnetici negli ambienti di lavoro, Ipsoa, 2008.

CASALE, D., "La prova del nesso di causalità professionale per le tecnopatie non tabellate. A proposito della prima pronuncia che riconosce gli effetti cancerogeni dei campi elettromagnetici dei telefoni cellulari”, en $A D L$, núm. 6., 2010.

CERATO, M., y ROMEO, M., "Inquinamento elettromagnetico", en CENDON, P., La prova e il quantum nel risarcimento del danno, Utet, 2013.

CERBONE, M., Il giudice e l'Inail nell'applicazione delle norme sulle malattie professionali, Olympus, núm. 34., 2014. 
COGGIOLA, N., "Il giudice e la statistica: attività lavorative, esposizione all'amianto ed asbestosi ovvero quando il numero di morti e malati fa la prova nel proceso", en GI, 2005 .

DE MATTEIS, A., "La prova della malattia multifattoriale. L'equivoco continua", en RIMP, Vol., 101, núm. 3., 2014.

ENRIQUES, L., “Come si forma un avvocato”, en Lavoce.info, 26 de septiembre de 2013.

FIORI, A., "La causalità nelle malattie professionali", en Atti del VI Convegno nazionale di medicina legale previdenziale, S. Margherita di Pula 18-20 ottobre 2006, Inail, 2006.

GIUBBONI, S., LUDOVICO, G., Y ROSSI, A., Infortuni sul lavoro e malattie professionali, Cedam, 2014.

GOBBA, F., MODENESE, A., ZANOTTI, G., "Effetti dei campi elettromagnetici nei lavoratori professionalmente esposti: le evidenze scientifiche, le decisioni della magistratura", en GOLDONI, S., CONTESSA, G.M., NATALETTI, P., DELLA VECCHIA, N., y NICOLINI, O., Radiazioni ionizzanti e non ionizzanti: valutazione e protezione alla luce della nuova normativa europea, Modena, dBA, 2017, pp. 140-141, disponible en acceso abierto en la library de la plataforma SALUS, en la dirección http://salus.adapt.it.

GRECO, M., "Inquinamento elettromagnetico e salute fra controversie, ricerca indipendente ed associazionismo Il caso del movimento degli elettrosensibili”, en EtnoAntropologia, Vol. 4, núm 2., 2016.

LAGORIO, S., y VECCHIA, P., "Una Corte italiana riconosce l'origine professionale di un neurinoma del trigemino in un utilizzatore di telefoni mobili: un esempio concreto dei complessi rapporti tra scienza e diritto", en La Medicina del Lavoro, 2011, 102.

LEROUGE, L., "Réflexions juridiques autour du rapport "Santé au travaul: vers un systéme simplifié pour une prévention rendorcée"”, en Droit Social, 2019.

LEVIS, A.G., GRASSO, G., PALMISANO, S., CONSIGLIERE, F., y GENNARO, V., "A proposito dell'articolo su Med. Lav., 2011, 102, 144-162 di S. Lagorio e P. Vecchia. Telefoni mobili e tumori alla testa: la sentenza della Corte d'Appello di Brescia Sezione Lavoro - alla luce delle attuali conoscenze scientifiche e della legislazione in materia”, en La Medicina del Lavoro, núm. 103., 2012, pp. 309-313.

LEVIS, A.G., MINICUCI, N., RICCI, P., GENNARO, V., CROSIGNANI, P., y GARBISA, S., "Telefoni mobili e tumori alla testa: è tempo che i dati corretti vengano messi in evidenza e valorizzati?”, en Epidemiologia \& Prevenzione, 2011, pp. 188-199. 
MANCA, G., “Assalti e difese ai bastioni della causalità scientifica nei contributi più recenti di dottrina e giurisprudenza", en $R C P, 2013$, pp. 476-503.

MOCCALDI, R., y POLICHETTI, A., "Mancato riconoscimento come malattia professionale di un tumore alla parotide in esposto a campi elettromagnetici da telefoni mobili e a radiazioni ionizzanti”, en Aggiornamenti di Radioprotezione, 2016, pp. 4-19.

MONTUSCHI, L., e INSOLERA, G., Il rischio amianto. Questioni sulla responsabilità civile e penale, Bononia University Press, 2006.

PAOLINI, C., "Danni alla salute per uso abnorme del cellulare: si tratta di tecnopatia se sussiste il nesso causale della probabilità qualificata", en $A D L$, núm. 4-5, 2017.

RADOS, B. y GIANNINI, P., La consulenza tecnica nel processo civile, Giuffrè, 2013.

RIVERSO, R., "Dalla tutela del lavoratore alla tutela della persona. Il profilo oggettivo", en RIMP, 2015.

ROMEO, L., "Nesso di causalità nelle malattie multifattoriali", en RIMP, 2016.

ROTA, A., "Sulla natura professionale del tumore contratto per overuse del cellulare in ambito lavorativo", en RIDL, núm. 3., 2013.

SALOMONE, E., "Sulla motivazione con riferimento alla consulenza tecnica d'ufficio", en RTDPC, 2002.

SFERRA, C., "Confronto tra malattia professionale non tabellata e malattia comune", en RIMP, 2010.

TARUFFO, M., "Le prove scientifiche nella recente esperienza statunitense”, en RTDPC, 1996, pp. 219-249.

TRENTA, G., "Competenze e professionalità nelle consulenze d'ufficio in tribunale", en $L a$ Medicina del Lavoro, Vol. 102, núm. 2., 2011, pp. 163-166.

TULLINI, P., “A rischio amianto?”, en RIDL, vol. 4., 2007.

VARVARESSOS, A., "Riscontro epidemiologico e probabilità qualificata: nuove prospettive per l'accertamento in concreto dell'elemento causale nelle ipotesi di malattie non tabellate o ad eziologia multifattoriale”, en Rivista Italiana di Medicina Legale, núm., 2013.

VISCOMI, A., “Amianto: precauzione, prevenzione e responsabilità", en MONTUSCHI, L., e INSOLERA, G., Il rischio amianto. Questioni sulla responsabilità civile e penale, Bononia University Press, 2006. 
Las directrices de la jurisprudencia italiana sobre enfermedades profesionales derivadas de la exposición...

ZANETTI, R., "Il sapere scientifico in tribunale", en Epidemiologia e Prevenzione, 2005, pp. 307-309. 